\title{
Obesity, knee osteoarthritis, and polypathology: factors favoring weight loss in older people
}

\author{
This article was published in the following Dove Press journal: \\ Patient Preference and Adherence \\ 27 May 2016 \\ Number of times this article has been viewed
}

\author{
Pilar Isla Pera' \\ Ma Carmen Olivé Ferrér \\ Montserrat Nuñez Juarez ${ }^{2}$ \\ Esther Nuñez Juarez ${ }^{3}$ \\ Loreto Maciá Soler ${ }^{4}$ \\ Carmen López Matheu' \\ Assumpta Rigol Cuadra' \\ María Honrubia Pérez' \\ Diana Marre 5 \\ 'School of Nursing, University \\ of Barcelona, ${ }^{2}$ Department of \\ Rheumatology, Musculoskeletal Clínic \\ Institute Hospital Clínic, ${ }^{3}$ Institut \\ Català de la Salut, Barcelona, ${ }^{4}$ Nursing \\ Department, University of Alicante, \\ Alicante, ${ }^{5}$ Universitat Autònoma de \\ Barcelona, Barcelona, Spain
}

Correspondence: Pilar Isla Pera School of Nursing, University of Barcelona, Feixa Llarga s/n L'Hospitalet de Llobregat, Barcelona 08907, Spain Tel +34934024 24l Email pisla@ub.edu
Aim: We aimed to explore the meaning of obesity in elderly persons with knee osteoarthritis (KO) and to determine the factors that encourage or discourage weight loss.

Background: Various studies have demonstrated that body mass index is related to $\mathrm{KO}$ and that weight loss improves symptoms and functional capacity. However, dietary habits are difficult to modify and most education programs are ineffective.

Design: A phenomenological qualitative study was conducted. Intentional sampling was performed in ten older persons with $\mathrm{KO}$ who had lost weight and improved their health-related quality of life after participating in a health education program. A thematic content analysis was conducted following the stages proposed by Miles and Huberman.

Findings: Participants understood obesity as a risk factor for health problems and stigma. They believed that the cause of obesity was multifactorial and criticized health professionals for labeling them as "obese" and for assigning a moral value to slimness and diet. The factors identified as contributing to the effectiveness of the program were a tolerant attitude among health professionals, group education that encouraged motivation, quantitative dietary recommendations, and a meaningful learning model based on social learning theories.

Conclusion: Dietary self-management without prohibitions helped participants to make changes in the quantity and timing of some food intake and to lose weight without sacrificing some foods that were deeply rooted in their culture and preferences. Dietary education programs should focus on health-related quality of life and include scientific knowledge but should also consider affective factors and the problems perceived as priorities by patients.

Keywords: obesity, elderly, polypathology, therapeutic education, diet, nursing, knee osteoarthritis

\section{Introduction}

Knee osteoarthritis (KO) is one of the most important causes of disability among the elderly, while obesity is a risk factor for numerous chronic diseases and forms of cancer. ${ }^{1}$ In Spain, the prevalence of KO is $10.2 \%$ in persons older than 20 years and is $30 \%$ in those older than 60 years. ${ }^{2}$ The prevalence of overweight and obesity in the Spanish population is $25 \%$ and is $84 \%$ in persons older than 65 years. ${ }^{3,4}$

Various studies have demonstrated that body mass index (BMI) is related to $\mathrm{KO}^{5}$ and influences its progression ${ }^{6}$ and that weight loss in obese $\mathrm{KO}$ patients slows disease progression and improves symptoms and functional capacity. ${ }^{7}$ However, dietary habits are difficult to modify and most education programs are ineffective. ${ }^{8,9}$

\section{Background}

Although it is widely accepted that obesity is a complex, multifactorial, chronic disease whose development involves the interplay between genetic and environmental factors, most studies have been conducted from medical or psychological perspectives. 
From the medical point of view, the main cause of overweight and obesity is an imbalance between calorie intake and expenditure, which translates into energy storage in the form of fat. ${ }^{10}$ From the psychological perspective, there is a positive association between obesity and certain psychological factors, such as anxiety, depression, and stress. ${ }^{11}$ Some studies have reported an association between weight gain and the onset of depression; ${ }^{12}$ however, the opposite has been found in countries with a different culture. ${ }^{13}$ Also important are studies that consider food as an addictive substance, although a diagnosis of food addiction can be made in persons who are of normal weight or are underweight. ${ }^{14}$ These types of studies do not consider the socioeconomic, political, or cultural causes of obesity and consequently the strategies they discuss for its prevention or treatment focus on biological factors or the individual, as they consider obesity to be caused mainly by the person. However, despite the concern generated by overweight and obesity, as well as the interventions and recommendations for its prevention, the global obesity epidemic continues to increase and it has been estimated that the mean BMI has risen by $0.4 \mathrm{~kg} / \mathrm{m}^{2}$ per decade since $1980 .{ }^{15}$

Criticisms of the weight-centered approach began to be voiced in the 1990s because most people are unable to maintain their initial weight loss in the long term and therefore dietary treatment is unlikely to be effective in reducing obesity-associated morbidity and mortality. ${ }^{16}$ Other studies show that the weight-centered approach can provoke eating disorders and an excessive concern with body image, leading to low self-esteem and stigma. ${ }^{17}$ These findings have led to an increase in qualitative studies and social anthropology approaches in the past few years with the aim of providing a complementary knowledge and more comprehensive approach to the problem.

The results of qualitative studies show that overweight and obesity are complex phenomena with multiple causes and involve the interplay of personal, socioeconomic, cultural, and psychological factors. ${ }^{18-20}$ The social sciences perspective has criticized the moral burden placed on obese persons and stresses that eating does not simply satisfy a biological need, as proposed by the mechanistic view of the body, but also involves social, psychological, economic, and cultural factors. ${ }^{21}$ For sociology, overweight and obesity reflect the social and gender inequalities in modern society. The association between poverty and obesity has been widely documented, with the poorest people being those most affected by overweight and obesity. ${ }^{22}$ Obesity is associated with lower socioeconomic status and educational level in women, ${ }^{23}$ while this association is less clear in men. ${ }^{24}$

Considering the problem posed by obesity and $\mathrm{KO}$ in elderly patients requiring total knee replacement, in 2012, a therapeutic education and functional readaptation (TEFR) program was conducted in a group of 45 patients in a tertiary hospital. This 4-month program consisted of two 40-minute individual visits and three 90-minute group sessions. The program was designed following the methodology established for this type of program ${ }^{25}$ and was based on social learning theories. ${ }^{26}$ The program was conducted by a nurse with the support of a dietitian. The mean age of the participants was $67.11 \pm 7.9$ years; the mean BMI was $40.47 \pm 4.22 \mathrm{~kg} / \mathrm{m}^{2}$, and the mean number of comorbidities was 7.02 \pm 3.08 . Most of the patients $(93 \%)$ were women. In all patients, the program reduced pain and body weight and improved health-related quality of life (HRQoL).

The results of the program led to the following research questions: what meaning do elderly persons with polypathology assign to obesity? Which factors encourage or discourage weight loss?

\section{Aim}

The aim of this study was to explore the meaning assigned by elderly individuals with polypathology to obesity and to identify, from the patients' perspective, the factors that encourage or discourage weight gain and weight loss.

\section{Design}

A qualitative phenomenological study was performed, using symbolic interactionism ${ }^{27}$ as the theoretical framework, as this theory assigns great importance to the ability of individuals to interpret the social world.

\section{Sample and participants}

We included ten patients who had participated in the TEFR program. The participants were selected according to their age, sex, profession, and educational level to ensure the representativeness of the sample. The informants' characteristics are shown in Table 1.

\section{Data collection}

A focus group (FG) meeting was held in 2013 with the ten selected patients. This technique was used because the TEFR program combined individual and group sessions and the aim was to identify not only individual opinions but also to construct a collective reflection on the problem 
Table I Sociodemographic and clinical characteristics of patients

Sex

Male, $\mathrm{n}(\%) \quad 2(20)$

Female, $\mathrm{n}(\%)$

Mean age (years) \pm SD

$8(80)$

Educational level

None, $\mathrm{n}(\%)$

$67.23 \pm 7.87$

Primary ( $<5$ years), $\mathrm{n}(\%)$

Secondary ( $<10$ years), $\mathrm{n}(\%) \quad 3(30)$

Higher (> 10 years), $n(\%) \quad$ I (I0)

Occupational activity

Housewife, $\mathrm{n}(\%) \quad 2(20)$

Retired, $\mathrm{n}(\%)$

Mean number of comborbidities, mean \pm SD

$8(80)$

$7.02 \pm 3.08$

$40.47 \pm 4.22$

$92.35 \pm 8.93$

Mean weight at the start of the program, $\mathrm{kg}$

Mean weight at the end of the program

(4 months), $\mathrm{kg}$

$89.10 \pm 8.70$

$(P=0.003)$

of obesity. The FG lasted 2 hours and ended when data saturation had been reached. The FG was structured in five phases: 1) meaning of obesity, 2) the experience of being obese, 3 ) perceptions of the health care received, 4) factors encouraging or discouraging weight gain or weight loss, and 5) feedback and confirmation that the group was in agreement with the data collected. We also used the comments of the informants made before or after the FG, as well as the field notes made by an observer during the session and by the interviewer, at the end of the session. Sociocultural, demographic, and clinical data were collected from the medical records of each patient.

\section{Data analysis}

Data analysis was conducted by manual thematic analysis ${ }^{28}$ through an inductive approach but without losing sight of the aims of the study, following the stages proposed by Miles and Huberman. ${ }^{29}$ An exhaustive reading was made of the transcription of all the data. A list of the significant elements identified and the relationships among them was drawn up until a list of themes was obtained, grouped into categories.

\section{Rigor}

To strengthen the validity of the study, the data analysis was conducted by two researchers and was discussed with the rest of the team. The results are presented as narratives and supported by textual fragments of the participants' discourse to illustrate and confirm the relationship between the theoretical concepts and the data. The interviewer made an effort to acknowledge her assumptions about the phenomenon under study and to take into account the potential impact of her subjectivity on the data.

\section{Ethics considerations}

This project was approved by the Ethics Committee for Clinical Research of the Musculoskeletal Clinic Institute Hospital Clinic, Barcelona, Spain. Written informed consent was obtained from all participants, and data confidentiality was guaranteed.

\section{Findings}

Six themes were identified.

\section{Meaning of obesity}

Participants interpreted obesity as a physical characteristic producing a negative body image and as a risk factor that can cause or exacerbate diseases. They defined obesity using words such as ugliness, misfortune, torture, and social rejection. However, they believed that both slimness and obesity are social constructs linked to pressure from the media and fashion, indicating that they had achieved a critical and reflective distance from sociocultural images, an ability that some authors have described as social agency, ie, the capacity to follow or reject cultural mandates. ${ }^{30}$

I'd define obesity as a Chinese torture that makes one suffer because being slim is fashionable and if you aren't, everyone thinks less of you. [Juana]

$\mathrm{KO}$ was directly related to obesity but also to excess physical work and to the aging process. This disease was the main health problem perceived by participants due to its associated pain and functional limitations, but the osteoarthritis did not affect the knee alone but rather the whole body.

The worst thing is the osteoarthritis, it hurts me, it hurts my body, but it isn't just because of the obesity, because I've always had to work hard and, besides, it's an age thing and that can't be changed. [Luisa]

In addition to obesity and $\mathrm{KO}$, all the participants had comorbidities requiring dietary treatment but believed that these problems were normal for their age and difficult to resolve. Medical recommendations for risk reduction were accepted and formed part of their cultural repertoire, which translated into a repetition of medical discourse on risk factors and chronic diseases but not into significant lifestyle changes or, if these did occur, they did not persist in the long term. 
Yes, obesity can lead to complications in diabetes, heart problems, bone problems, but ... [Carlos]

\section{Causes of obesity}

Women tended to report that onset of obesity occurred with changes in the life cycle, particularly pregnancy and menopause, while men associated it with stressful events such as accidents or unemployment. Nevertheless, as in other studies, ${ }^{18-20}$ all informants believed that obesity was caused and perpetuated by multiple factors, including genetics and metabolic changes, as well as social, environmental, and psychological factors such as family eating patterns, the traditions of the social group, the media, and, in particular, stressful situations that prompt the use of food to reduce the tension provoked by worries, sadness, and daily frustrations.

When I was 8 years old, my father got cancer and I think that was when I began to eat more than I should have, because of the stress I was feeling. Lots of things have happened to me and I think that's why I gained weight. [Rosa]

I'm like this because of a car accident, I had to retire and I began to put on weight and also because, after the accident and because of it, they pumped me full of cortisone. [Luis]

Unlike some studies showing that obese persons attribute the main cause of obesity to internal factors, ${ }^{31}$ in this study, participants believed that obesity was triggered by external factors. Even a lack of interest or motivation could be caused by social and health problems provoking anxiety or stress, due to stigma that lowered self-esteem and the lack of family and societal support.

Now I actually do the exercises they've taught me, I watch what I eat and I've lost weight but before I was too worried by my health problems, problems at work and by everything and I didn't even want to go to the doctor. [Carlos]

\section{Psychosocial consequences of obesity}

Although the participants had achieved a reflective and critical distance from sociocultural images of obesity, this did not prevent most of them from feeling guilty, blamed, and discriminated against socially. Basdevant ${ }^{32}$ states that obese persons are considered nutritional delinquents for having failed at traditional diets and for not undertaking physical activity.

My son told me "Go look at yourself in the mirror and see how fat you are, see if that makes you take it seriously for once". This hurt me and made me feel ashamed, but I think that a shock like that is a good thing, so that you really take stock. [María]

What most worried the participants was not obesity but the social consequences of the condition, which were evident in all areas of their lives. The difficulty of finding clothes they liked at reasonable prices, especially among women, was a barrier to social relationships and, in some cases, family relations, provoking shame and humiliation.

I always look at what other people are wearing and it makes me angry that I can't wear the same things, so I don't go out much. That affects my relationships with other people and my partner. [Rosa]

Body image is influenced by the perception of body size and by people's attitudes and feelings toward their bodies, ie, their positive or negative assessment of their body image. ${ }^{33}$

I was putting on weight but I didn't see myself as fat, just chubby. But one day I went into a clothes shop and the sales assistant told me, rudely, "we don't have any sizes for you" and since then, I've begun to see myself as fat and to have a much worse image of myself. [Laura]

Some authors believe that one of the most important effects of obesity is the low self-esteem it produces in many affected individuals ${ }^{34}$ and that that is a risk factor for the development of eating disorders, coping strategies, and maintaining a perception of self-sufficiency. ${ }^{35}$

They say that fat people are more cheerful than slim ones, which is like a defense mechanism to please other people. I don't think so. Other people don't like you and I don't like myself either. [Laura]

In two participants, their obesity did not limit their lives or prevent them from enjoying it. They attributed this to family support and professional success, which gave these participants financial resources and independence. People with good self-esteem and self-concept reported they had good social recognition and satisfactory social relationships. In contrast, low self-esteem and self-concept were associated with a perception of social rejection and difficulties in social relationships. Self-esteem was also associated with perceived body image. Persons with good self-esteem accepted their obese bodies, although they would have liked to lose weight to feel better, while those with low self-esteem rejected their obese bodies and would have liked to have changed it to improve their personal image and feel more accepted by family and friends. 
I've always been fat and it's never stopped me from doing anything. I've played basketball, I go swimming. I've had a good job, friends, and a husband who has always supported me. I'd like to lose weight because it would be good for me. [Luisa]

Some people are really offensive, because the moment they see me eating they say: "later you'll say you want to lose weight". What do they know about how much I've eaten all day? This makes me suffer and that's why I'd like to lose weight. [Montserrat]

Although studies have reported contradictory results, a review $^{36}$ published in 2011 in Spain shows that most people with overweight or obesity have some type of psychopathological comorbidity.

\section{Health professionals' discourse}

The meanings of health and disease are social constructs that share scientific knowledge and sociocultural representations that are associated and show a reciprocal influence. For Contreras, ${ }^{37}$ in the case of obesity, the combination of the medical model and the cultural (aesthetic model) gives rise to the medical-cultural model, that is, the cultural model has been appropriated by medicine for health reasons. Many studies, eg, that by Apfeldorfer, ${ }^{38}$ have shown that health professionals play an important role in the process of stigmatizing persons with obesity.

Patients criticize health professionals for labeling them as "obese", assigning a moral value to slimness and diet and for seeing obesity as a characteristic linked to personal identity.

I go to one doctor after another and the referral note always says the same. It's how they present me; “Obese woman, obese woman". Shit! It's like you have the plague.

[Juana]

This is related to concepts of stigma and the moral career of stigmatized individuals, elaborated by Goffman. ${ }^{39}$ The term "moral career" denotes the transition from being a normal person to a stigmatized person. In some cases, the patient identifies with the disease (obesity), leading him or her to create an impaired identity, which, at first, establishes difference, then inferiority, and finally blaming of those labeled as different by their stigma. ${ }^{40}$

Although knowledge does not change dietary behavior, ${ }^{41}$ health professionals simplify the complex act of eating with repetitive recommendations on calorie reduction, prohibition of some foods, increased physical activity, and the threat of possible health complications, without bearing in mind that patients have heard the same discourse on multiple occasions and are well aware of the principles of healthy eating and the health risks of not following recommendations.

I believe that people expect more from their doctor. You go to the doctor and he tells you "Ah well, you have to lose weight" and that's it. They treat you like you were irresponsible and don't live healthily. [Carlos]

\section{Factors hampering adherence to recommendations}

Participants believed that there were multiple factors that reduced motivation and hampered lifestyle changes but perceived that the most important factors were the biological approach to obesity, not expecting to achieve a socially acceptable body, and state of mind.

The interventions conducted by the health system view obesity as a disease and medicalize food. ${ }^{42}$ Dietary recommendations based on immutable international guidelines predominate, which are generally applied dogmatically, independently of the sex or cultural group to which the obese person belongs.

I think that the cause of obesity is individual and then, each person decides if the sacrifice is worth it or not. The same recommendations don't work for everyone. [Luis]

However, participants did not see obesity as a disease and therefore its cure was not the main aim of weight loss. Most wanted to lose weight to achieve an acceptable body and reduce pressure from the social and health settings as well as reducing obesity-associated stigma. This was the main reason for starting a reduced calorie diet, and the reason for abandoning it was a lack of short- and long-term results. The successive experiences in terms of the difficulty of achieving one's goals and the rebound effect of subsequent weight gain increased stress or anxiety and led to greater food intake, with the consequent feelings of guilt, lack of self-efficacy, and reduction in self-esteem and self-concept.

Every time I tried to lose weight, I got fatter. I really deprived myself and lost a bit of weight, but when I stopped one day, out of frustration, I gained all the weight lost and put on even more than when I started. [Montserrat]

An essential factor for maintaining motivation is state of mind, which is influenced by the patient's subjectivity and by social and family reinforcement, as well as by the attitude of health professionals. 
If I go to the doctor and he tells me that I haven't lost weight and that I'm not following the diet properly, I get discouraged, and if I think positively, I'm able to follow the diet, take exercise, but if I wake up feeling down, I eat and spend all day in bed. [Luisa]

Advertising and pressure to be slim lead many patients to attend private centers that promise rapid weight loss and provide a less demanding and blaming relationship than that offered by health professionals in the public sector. However, all participants attending these centers felt misled, due to the high cost and failure to meet expectations.

I attended two sessions a week, they recommended pills and a whole ton of complementary products and it was all "buy this and that", it was like throwing money into a bottomless pit. [Luis]

Public acceptance of the medical-cultural model and the moral value assigned to slimness and diets have led to the growth of the market for products, techniques, and false solutions. Importantly, the body represents a prime area of consumption and, at the same time, is one of the most frequently commercialized objects. ${ }^{43}$

\section{Factors encouraging a change of lifestyle}

For patients, the factors encouraging a change of lifestyle were support and a tolerant and respectful attitude among health professionals, active and meaningful methods based on social learning theories, group education encouraging motivation, autonomy in dietary self-management, and goals prioritizing perceived health problems.

In this study, all participants felt they received professional warmth and support. Social support is positively related to the process of health and disease and, in chronic diseases, the most important form of assistance is emotional support. $^{44,45}$

What I most value is how they treat you, that you see that they are looking after you and are on your side.

[Montserrat]

Another source of motivation is the perception that medical attention is aimed at reducing pain and improving functionality and HRQoL, which are priorities for most patients. These results are in line with some initiatives aiming to improve HRQoL rather than promote weight loss. One example is the Health at Every Size (HAES) initiative (ASDAH, 2015), ${ }^{46}$ which supports the promotion of healthy behavior independently of weight loss. Although further studies are required to provide scientific evidence supporting this paradigm, ${ }^{47}$ some studies have shown that it produces clinical and psychological improvements. ${ }^{48}$ In addition, the World Health Organization and the national strategies of most countries acknowledge the need to include the three dimensions (biological, symbolic, and social) of eating in their recommendations. Unfortunately, many of these recommendations are purely theoretical, with limited direct application in clinical practice.

For me, the most important thing is to be in less pain and be able to move. People respect you and when they see you've put on a bit of weight, they say "don't worry, it doesn't matter", the important thing is that you feel good. [Montserrat]

Another factor identified by patients as motivating is active-and-meaningful learning through a format combining individual and group sessions.

If I had to give it a mark, I'd give it a 10. Besides listening to you, they don't explain things by telling them to you but by doing them and that's what helps you to learn and reflect on how you do things. [María]

Education imparted during individual sessions allowed patients to explain their personal experiences, perceptions, and intimate emotions, which would be difficult to explain in a group and gave them the feeling that the attention was adapted to their individual needs.

I think it's good to be alone with the nurse because there are some things you're not going to tell everyone and you feel that the time is just for you. [Luisa]

Group education, as described by Levine and Perkins, ${ }^{49}$ promotes the feeling of belonging to a social group; it allows people to assign meaning to particular circumstances and to people who do not fit into the prescribed ideal; it aids awareness of the situation, provides models for behavior and reciprocal help, as well as providing a social network that reduces isolation and social stigma. The psychoeducational group also aids the application of Bandura's ${ }^{26}$ social learning theory, which holds that human behavior is largely a result of modeling. Exchanging experiences with people with similar problems facilitates a change in attitudes and the learning of new stress management techniques and social skills.

I prefer the group, because you think that you're the only one with problems and you see that a lot of people are 
like you and the group encourages you and motivates you because someone says something and you can have an opinion and learn from others. Individually, we all feel that everything hurts and we're all carrying too much weight but in the group you don't just explain what's happening to you, everyone says something and you can learn and understand better what's happening to you. [Juana]

The most valued aspect of the program was that it did not provide a standard diet nor did it prohibit any type of food. Considering that people have knowledge of food composition, they should have the autonomy to selfregulate the quantity and timing of food intake freely and responsibly.

The other programs told you not to eat this or that and you were afraid to go back if you hadn't lost weight and now they tell you that you can eat everything but you yourself have to control the amount. A bit of chocolate? Of course, why not? Paella? That's OK too. You make up the diet every day and that's very motivating. [Laura]

Dietary habits in adults form routine patterns of consumption that are constituted throughout life and are highly rooted in the members of a culture. Their stability, or resistance to change, is more pronounced in older persons. ${ }^{50}$ The autonomy to self-regulate one's own food intake is important because elderly individuals who manage their own lives also have greater self-esteem than those who feel controlled by others. ${ }^{51}$ A recent systematic review ${ }^{52}$ also showed that the psychological factors most predictive of a change in dietary habits were the perception of self-efficacy and the degree of autonomy, as well as the capacity for self-regulation.

\section{Discussion}

The results of this study are similar to those of other qualitative studies in adults concerning the meaning of obesity and its psychosocial consequences and are also in line with initiatives arguing that dietary education should focus on HRQoL and not weight loss. ${ }^{47,48}$ Some differences are that older persons in this study identified external factors as the main triggers of obesity and had a critical attitude to sociocultural models imposing a "healthy" and prescribed body image, although this did not prevent these patients from also feeling blamed and stigmatized. Similar to other studies, ${ }^{53}$ this study found that none of the patients felt satisfied with having a large body. However, differences were found in the motives for losing weight, which were strongly related to the perception of body image and self-esteem.
As in other studies, autonomy increased the perception of quality of life $\mathrm{f}^{54}$ and the support of health professionals and educational methodology were factors that favored a change in behavior and HRQoL. ${ }^{55-57}$ Although there is little evidence on the most effective educational methods, many studies ${ }^{58-60}$ agree on the benefits of group and individual education versus individual education alone. Although patients attended a single hospital, we believe that the results obtained could be transferred to other contexts, because older people have similar health problems and the cultural problems of obesity are a global phenomenon.

\section{Limitations}

Data were collected a year after the program ended, which could have led to some information or emotions being forgotten but allowed confirmation that participants had maintained weight loss and dietary changes. No individual interviews were conducted because it was thought that the informants would already have a collective construction of the problem of obesity after their previous participation in group sessions. Nevertheless, in the group interview, participants were encouraged to express individual differences of opinion.

\section{Conclusion}

Older people have created a reflective and critical distance from sociocultural images of obesity but nevertheless feel guilty, blamed, and discriminated against socially. These patients feel stigmatized and pressurized - mainly by health professionals and, by extension, their families - to achieve a "healthy", socially acceptable body.

Self-esteem influences goals, the perception of selfefficacy, and motivation. Protective factors against negative discourse about obesity are family support, social networks, and professional satisfaction.

Dietary self-management without prohibitions increases the perception of autonomy and self-esteem and allows weight loss without having to sacrifice certain foods that are deeply rooted in the older person's culture and preferences.

Patients need to have realistic expectations focused on improving health and HRQoL and not on achieving a "socially prescribed" body. The support of nursing professionals and educational methods are essential to promote self-esteem and to reduce stigma and social isolation.

\section{Disclosure}

PIP, MCOF, CLM, and ARC are members of the Research Adoptions, Families, Childhoods group, with DM as the Principal Investigator. MNJ is a member of the Health Outcomes 
in Musculoskeletal Pathologies (RESPAL) and Plasticity Institut d'Investigacions Biomèdiques August Pi i Sunyer groups. ENJ is a member of the RESPAL group. The authors report no other conflicts of interest in this work.

\section{References}

1. Catenacci VA, Hill JO, Wyatt HR. The obesity epidemic. Clin Chest Med. 2009;30:415-444.

2. Nuñez M, Nuñez E, Sastre S, Del-Val JL, Segur JM, Macule F. Prevalence of knee osteoarthritis and analysis of pain, rigidity, and functional incapacity. Orthopaedics. 2008;31(8):753.

3. Berghöfer A, Pischon T, Reinhold T, Apovian CM, Sharma AM, Willich SN. Obesity prevalence from a European perspective: a systematic review. BMC Public Health. 2008;8:200-210.

4. Gomez-Cabello A, Pedrero-Chamizo R, Olivares PR, et al; EXERNET Study Group. Prevalence of overweight and obesity in noninstitutionalized people aged 65 or over from Spain: the elderly EXERNET multi-centre study. Obes Rev. 2011;12(8):583-592.

5. Christensen R, Henriksen M, Leeds AR, et al. Effect of weight maintenance on symptoms of knee osteoarthritis in obese patients: a twelvemonth randomized controlled trial. Arthritis Care Res (Hoboken). 2015; 67(5):640-650.

6. Reijman M, Pols HA, Bergink AP, et al. Body mass index associated with onset and progression of osteoarthritis of the knee but not of the hip: the Rotterdam Study. Ann Rheum Dis. 2007;66(2):158-162.

7. Messier SP. Obesity and osteoarthritis; disease genesis and no pharmacological weight management. Rheum Dis Clin North Am. 2009; 93(1):145-159,xi-xii.

8. Phillips K, Wood F, Kinnersley P. Tackling obesity: the challenge of obesity management for practice nurses in primary care. Fam Pract. 2014;31(1):51-59.

9. Fillingham A, Peters S, Chisholm A, Hart J. Early training in tackling patient obesity: a systematic review of nurse education. Nurse Educ Today. 2014;34(3):396-404.

10. Pereira-Lancha LO, Campos-Ferraz PL, Lancha AH Jr. Obesity: considerations about etiology, metabolism, and the use of experimental models. Diabetes Metab Syndr Obes. 2012;5:75-87.

11. Ziauddeen H, Subramaniam N, Gaillard R, Burke LK, Farooqi IS, Fletcher PC. Food images engage subliminal motivation to seek food. Int J Obes (London). 2012;36(9):1245-1247.

12. Desai MD, Patoliya HM. Impact of body weight and perception of overall body image on depression among post-graduate female students. Indian J Community Psychol. 2011;7(2):341-346.

13. Chang H, Yen S. Association between obesity and depression: evidence from a longitudinal simple of the elderly in Taiwan. Aging Ment Health. 2012;16(2):173-180.

14. Pelchat M. Food addiction in humans. J Nutr. 2009;139(3):620-622.

15. Finucane MM, Stevens GA, Cowan MJ, et al; Global Burden of Metabolic Risk Factors of Chronic Diseases Collaborating Group (Body Mass Index). National, regional, and global trends in body-mass index since 1980: systematic analysis of health examination surveys and epidemiological studies with 960 country-years and 9.1 million participants. Lancet. 2011;377(9765):557-567.

16. Garner DM, Wooley SC. Confronting the failure of behavioral and dietary treatments for obesity. Clin Psychol Rev. 1991;11:729-780.

17. Bacon L, Aphramor L. Weight science: evaluating the evidence for a paradigm shift. Nutr J. 2011;24(10):9.

18. Barlösius E, Philipps A. Felt stigma and obesity: introducing the generalized other. Soc Sci Med. 2015;130:9-15.

19. Ferrari M. Understanding the feasibility of integrating the eating disorders and obesity fields: the beyond obesity and disordered eating in youth (BODY) Study. Eat Weight Disord. 2015;20(2):257-269.

20. Craft JM, Ridgeway JL, Vickers KS, Hathaway JC, Vincent A, Oh TH. Unique barriers and needs in weight management for obese women with fibromyalgia. Explore (NY). 2015;11(1):51-58.
21. Vigarello G. The healthy and unhealthy. Madrid: Abadía Lectores; 2006.

22. Keating C, Backholer K, Gearon E, et al. Prevalence of class-I, class-II and class-III obesity in Australian adults between 1995 and 2011-2012. Obes Res Clin Pract. 2015;9:553-562.

23. Flegal KM, Carroll MD, Ogden CL, Curtin LR. Prevalence and trends in obesity among US adults, 1999-2008. JAMA. 2010;303(3): 235-241.

24. Paeratakul S, Lovejoy JC, Ryan DH, Bray GA. The relation of gender, race and socioeconomic status to obesity and obesity comorbidities in a sample of US adults. Int J Obes Relat Metab Disord. 2002;26(9): $1205-1210$.

25. Nuñez M, Nuñez E, Segur JM, et al. The effect of an educational program to improve health-related quality of life in patients with osteoarthritis on waiting list for total knee replacement: a randomized study. Osteoarthritis Cartilage. 2006;3:279-285.

26. Bandura A. Social Learning Theory. Madrid: Espasa-Calpe; 1984.

27. Blumer H. Symbolic interactionism. Perspective and method. Barcelona: Spain; Hora; 1982.

28. Minayo MC. Qualitative analysis: theory, steps and reliability. Cien Saude Colet. 2012;17(3):621-626.

29. Miles M, Huberman M. Qualitative Data Analysis: An Expanded Sourcebook. Newbury Parck, CA: Sage; 1994.

30. Contreras Hernandez J, Grace M. Arnaiz Food and culture. Anthropological Perspectives. Barcelona: Ariel; 2005.

31. Niederdeppe J, Robert SA, Kindig DA. Qualitative research about attributions, narratives, and support for obesity policy. Prev Chronic Dis. 2011;8(2):A39.

32. Basdevant A. Purpose and abuses of contemporary medical definition. In: Csergo J, editor. Too big? Obesity and its representations. Paris: Éditons other; 2009:112-122.

33. Lokken KL, Boeka AG, Yellumahanthi K, Wesley M, Clements RH. Cognitive performance of morbidly obese patients seeking bariatric surgery. Am Surg. 2010;76:55-59.

34. Kalarchian MA, Marcus MD, Levine MD, et al. Psychiatric disorders among bariatric surgery candidates: relationship to obesity and functional health status. Am J Psychiatry. 2007;164(2):328-334.

35. Gasco M, Briñol P, Horcajo J. Attitude change toward body image: the role of elaboration on attitude strength. Psicothema. 2010;22(1):71-76.

36. Baile JI, Gonzalez MJ [webpage on the Internet]. Psychopathological comorbidity in obesity. Anales Sis San Navarra [magazine on the Internet]; 2011;34(2):253-261. Available in: http://scielo.isciii.es/scielo. php?script=sci_arttext\&pid=S1137-66272011000200011\&lng=es; http://dx.doi.org/10.4321/S1137-66272011000200011. Accessed March 8, 2015.

37. Contreras J. Obesity: a sociocultural perspective. Continuing Education in Nutrition and Obesity. 2002;5(6):275-286.

38. Apfeldorfer G. Lose weight, it's crazy, Paris: Odile Jacob; 2000.

39. Goffman E. Stigma. Deteriorated Identity. Buenos Aires: Argentina; Routledge publishers; 1998.

40. Porter R. The Faber Book of Madness. London: Faber \& Faber; 1991.

41. Martín Criado E. Nutrition-related knowledge scarcely leads to any eating habit changes. The case of working-class mothers in Andalusia, Spain. Revista Española de Salud Pública. 2007;81:519-528.

42. De Domingo-Bartolomé M, López Guzmán J. The medicalization of food. Bioethical Perspectives. 2014;18(2):170-183.

43. Martinez Hernaez A. The imaginary body form modernity. Debats. 2009;79:8-17.

44. Brooks AT, Andrade RE, Middleton KR, Wallen GR. Social support: a key variable for health promotion and chronic disease management in Hispanic patients with rheumatic diseases. Clin Med Insights Arthritis Musculoskelet Disord. 2014;7:21-26.

45. Kirk JK, Ebert CN, Gamble GP, Ebert E. Social support strategies in adult patients with diabetes. A review of strategies in the USA and Europe. Exp Rev Endocrinolol Metab. 2013;8(4):379-389.

46. Association for Size diversity and health (ASDAH) [webpage on the internet]. Available from: https://www.sizediversityandhealth.org/ index.asp. Accessed July 8, 2015. 
47. Penney TL, Kirk SF. The health at every size paradigm and obesity: missing empirical evidence may help push the reframing obesity debate forward. Am J Public Health. 2015;105(5):e38-e42.

48. Humphrey L, Clifford D, Morris MN. Health at every size college course reduces dieting behaviors and improves intuitive eating, body esteem, and anti-fat attitudes. J Nutr Educ Behav. 2015;47(4):354.e-360.e.

49. Levine M, Perkins DV. Principles of Community Psychology: Perspectives and Applications. Oxford: Oxford University Press; 1987.

50. Alvarez Munarriz L, Alvarez de Luis A [webpage on the Internet]. Lifestyles and food consumption. Gazeta de Antropología. 2009;25(1). [citado May 5, 2015]. Available from: http://www.ugr.es/ pwlac/ G25_27Luis_Alvarez-Amaia_Alvarez.html. Accessed March 8, 2015.

51. Zegers B. Performance and Capacity Intellectuals. In: Marin P, editor. New time for the Elderly. Santiago de Chile: Ediciones Universidad Catolica; 1993:133-150.

52. Teixeira PJ, Carraça EV, Marques MM, et al. Successful behavior change in obesity interventions in adults: a systematic review of selfregulation mediators. BMC Med. 2015;13(1):84.

53. ML Garza hair, JG Zuniga Zárate. Intrapersonal and family aspects associated with obesity: a phenomenological analysis. Science. 2007;10(2): 183-188.
54. Godwin M, Pike A, McCrate F, et al. Descriptive analysis by sex of cognitively functioning elderly patients. Can Fam Physician. 2015; 61(3):142-147.

55. Brooks J, King N, Wearden A. Couples' experiences of interacting with outside others in chronic fatigue syndrome: a qualitative study. Chronic Illn. 2014;10(1):5-17.

56. Trunzo J, Pinto B. Social support as a mediator of optimism and distress in breast cancer survivors. J Consult Clin Psychol. 2003;71:805-811.

57. Kirk S. Transitions in the lives of young people with complex healthcare needs. Child Care Health Dev. 2008;34(5):567-575.

58. Trento M, Passera P, Borgo E, et al. A 3-year prospective randomized controlled clinical trial of group care in type 1 diabetes. Nutr Metab Cardiovasc Dis. 2005;15(4):293-301.

59. Deakin TL, McShane CE, Cade JE, Williams RD. Group based training for self-management strategies in people with type 2 diabetes mellitus. Cochrane Database Syst Rev. 2005;18(2):CD003417.

60. Gucciardi EL, Demelo M, Lee RN, Grace SL. Assessment of two culturally competent diabetes education methods: individual versus individual plus group education in Canadian Portuguese adults with type 2 diabetes. Ethn Health. 2007;12(2):163-187.
Patient Preference and Adherence

\section{Publish your work in this journal}

Patient Preference and Adherence is an international, peer-reviewed, open access journal that focuses on the growing importance of patient preference and adherence throughout the therapeutic continuum. Patient satisfaction, acceptability, quality of life, compliance, persistence and their role in developing new therapeutic modalities and compounds to optimize

\section{Dovepress}

clinical outcomes for existing disease states are major areas of interest for the journal. This journal has been accepted for indexing on PubMed Central. The manuscript management system is completely online and includes a very quick and fair peer-review system, which is all easy to use. Visit http://www. dovepress.com/testimonials.php to read real quotes from published authors. 